\title{
A Narrative Review of Ultrasound Technologies for the Prediction of Neoadjuvant Chemotherapy Response in Breast Cancer
}

\author{
Jing Wang (D) \\ Yanhua Chu \\ Baohua Wang \\ Tianan Jiang $\mathbb{D}$
}

Department of Ultrasound, The First Affiliated Hospital, Zhejiang University School of Medicine, Hangzhou, Zhejiang, 310003, People's Republic of China
Correspondence: Tianan Jiang Department of Ultrasound, The First Affiliated Hospital, Zhejiang University School of Medicine, Hangzhou, Zhejiang, 310003 , People's Republic of China $\mathrm{Tel} / \mathrm{Fax}+8657187236628$

Email tiananjiang@zju.edu.cn

\begin{abstract}
The incidence and mortality rate of breast cancer (BC) in women currently ranks first worldwide, and neoadjuvant chemotherapy (NAC) is widely used in patients with BC. A variety of imaging assessment methods have been used to predict and evaluate the response to NAC. Ultrasound (US) has many advantages, such as being inexpensive and offering a convenient modality for follow-up detection without radiation emission. Although conventional grayscale US is typically used to predict the response to NAC, this approach is limited in its ability to distinguish viable tumor tissue from fibrotic scar tissue. Contrast-enhanced ultrasound (CEUS) combined with a time-intensity curve (TIC) not only provides information on blood perfusion but also reveals a variety of quantitative parameters; elastography has the potential capacity to predict NAC efficiency by evaluating tissue stiffness. Both CEUS and elastography can greatly improve the accuracy of predicting NAC responses. Other US techniques, including three-dimensional (3D) techniques, quantitative ultrasound (QUS) and US-guided near-infrared (NIR) diffuse optical tomography (DOT) systems, also have advantages in assessing NAC response. This paper reviews the different US technologies used for predicting NAC response in $\mathrm{BC}$ patients based on the previous literature.
\end{abstract}

Keywords: breast cancer, ultrasound, neoadjuvant chemotherapy

\section{Introduction}

The incidence and mortality rate of breast cancer (BC) in women currently ranks first worldwide, ${ }^{1}$ and based on current NCCN guidelines, patients with different molecular types of BC should undergo neoadjuvant chemotherapy (NAC) regardless of their primary stage or aim of surgery. ${ }^{2}$ NAC not only reduces tumor grade and improves patients' opportunities for surgery, but also allows new advances in cancer management by identifying new genetic pathways and drugs involved in cancer and improving patient survival. ${ }^{3-7}$ However, some patients receiving NAC may gradually develop resistance to drugs during the process of chemotherapy, which limits its clinical efficacy and leads to treatment failure. These patients would benefit from planning treatment and surgery methods by assessing the response to NAC considering new treatment methods. ${ }^{8}$ Chemotherapy can produce physiological and psychological side effects of varying degrees. Non-responders need to be accurately and promptly identified to avoid ineffective chemotherapy and side effects. ${ }^{9,10}$ As a result, accurately monitoring and evaluating the efficacy of NAC is becoming increasingly crucial. 
At present, the gold standard for evaluating the response to NAC is pathological assessment, ${ }^{11}$ and the relevant methods include the residual cancer burden score $(\mathrm{RCB})^{12}$ and Miller-Payne system. ${ }^{13}$ As pathological assessment requires breast tumor specimens after surgery, it is not suitable for dynamically monitoring the efficacy of NAC. In fact, imaging assessments are used primarily to monitor and evaluate the response to NAC. In addition, accurate imaging assessment of the residual tumor after NAC can provide important information for selecting the most appropriate surgical procedure and for predicting patient prognosis.

A variety of imaging assessment methods, including magnetic resonance imaging (MRI), computed tomography (CT), positron emission tomography (PET), ultrasound (US), mammography (MM) and so on, have been used to predict and evaluate the response to NAC. Each imaging assessment method has unique advantages and disadvantages according to the different imaging principles, expenditure and safety. Many studies maintain that MRI and PET exhibit higher accuracy in predicting the efficacy of NAC. ${ }^{14-18}$ Changes in tumor size are typically used to monitor the efficacy of NAC by palpation and imaging methods. According to Response Evaluation Criteria in Solid Tumors (RECIST) criteria, US is not suitable for monitoring tumor size due to high operator dependence and low reproducibility. ${ }^{19}$ However, with the development of new techniques, such as contrast-enhanced imaging, elastography, and 3D imaging, US is increasingly applied to predicting the efficacy of NAC. The objective of this paper is to review the role of US in monitoring the response to NAC in BC patients. According to a PubMed search, using the search terms "breast cancer", "neoadjuvant chemotherapy" and "ultrasound" and screening the search results, this paper reviews the previous literature and summarizes these studies.

\section{Grayscale US for Assessing Response to NAC}

US imaging is based on the conduction and reflection of high-frequency mechanical sound waves in the tissue, and the information of ultrasonic impulses and their reflections as echoes are converted and processed into real-time images. ${ }^{20}$ Grayscale US has been widely used to distinguish malignant from benign breast tumors, and it has also been applied to predict the response to NAC. Grayscale US is primarily applied to measure changes in tumor size after NAC. Most researchers have demonstrated that US underestimates breast tumor size. ${ }^{21-24}$ However, it was reported that US overestimates $\mathrm{BC}$ tumor size. Vriens et al examined 182 patients with $\mathrm{BC}$ and found that US overestimated size in $20 \%$ of patients. ${ }^{25}$ Whether US has an advantage in estimating tumor size compared with other imaging methods has not been determined. Stein et al measured 6543 primary $\mathrm{BC}$ patients and found that the correlation with histology was 0.61 for $\mathrm{MM}$ and 0.60 for US. $^{26}$ They demonstrated that the prediction of tumor size by US and MM was coincident. The result was the same as that of Chagpar et al study. ${ }^{27}$ However, Keune et al reported that US correctly measures residual tumor size in response to NAC more often than MM $(91.3 \%$ vs $51.9 \%){ }^{28}$ Both Vriens et $\mathrm{al}^{25}$ and Choi et $\mathrm{al}^{29}$ study compared the role of MRI and US in measuring tumor size and found that both were consistent in estimating size. The sensitivity, specificity, accuracy, positive predictive value (PPV), negative predictive value (NPV), falsenegative rates (FNR) and false-positive rates (FPR) were 36-61\%, 78-90\%, 71-74\%, 60-67\%, 29-75\%, 20-38\%, $35-60 \%$, respectively, for US-predicted remission in BC after NAC. ${ }^{30-35}$ The reason for the lower prediction efficiency might be due to the potential limitation of the ability of US to distinguish viable tumor tissue from fibrotic scar tissue. ${ }^{30-32}$

\section{Contrast-Enhanced Ultrasound (CEUS) for Assessing Response to NAC}

There is currently increasing awareness that anatomical approaches based on measuring tumor size have substantial limitations and that morphological changes often manifest later than functional changes. ${ }^{36}$ As a result, CEUS, dynamic contrast-enhanced MRI (DCE-MRI) and fluorodeoxyglucose positron emission tomography and computed tomography (FDG-PET/CT), which can quantify tumor functions, are playing increasingly larger roles in evaluating and predicting the response to NAC. CEUS might be one of the most direct imaging tools for visualizing perfusion changes in tumors. ${ }^{37}$ In fact, the development of contrast agents has improved the diagnostic accuracy of US over the past decade. ${ }^{38}$ Sonovue, a secondgeneration US contrast agent, has a microbubble diameter of 2 to $4 \mathrm{~mm}$. It can remain within the blood pool because it is close to the diameter of blood cells and cannot diffuse into the interstitial space. CEUS has been widely used in 
the ultrasonic diagnosis of diseases of various organs throughout the body. In recent years, CEUS has also been used to evaluate the efficacy of NAC, and it was demonstrated to be effective for assessing the response to NAC in BC patients compared to other methods. ${ }^{25,39}$ Previous studies showed that the sensitivity, specificity, accuracy, PPV, NPV, area under the curve (AUC), positive and negative likelihood ratios (LR+ and LR-) and diagnostic odds ratios (ORs) of CEUS imaging for predicting response to NAC were $85-96 \%, 78-87 \%, 84-86 \%, 92 \%$, $78 \%, \quad 0.71-0.92,4.49-5.5,0.15-0.16$ and $32.21-36$, respectively. ${ }^{16,40-44}$ Currently, CEUS is recommended for the evaluation of the efficacy of NAC and may be a promising tool for the evaluation of NAC response. ${ }^{45}$

Imaging may underestimate or overestimate the size of breast tumors, as mentioned above. It was found that the tumor sizes measured both before and after NAC by CEUS were larger than those measured by conventional grayscale US. ${ }^{40}$ Although CEUS has no significant advantage in the accuracy of tumor size measurement before $\mathrm{NAC}$, it has a higher correlation with pathological results after NAC, and the measurement accuracy is higher than that of other imaging methods. ${ }^{45}$ In addition, necrosis at the tumor center was found to be detected early by CEUS, which showed a local blood perfusion defect, whereas conventional grayscale US was unable to detect whether there was necrosis unless there was a fluid area. ${ }^{40}$

The TIC of CEUS reflects the speed and quantity of the contrast agent in the tumor microvasculature. TIC analysis is a state-of-the-art technique for CEUS video quantification that contains a variety of blood perfusion quantitative parameters. $^{40,46,47}$ The quantitative parameters of blood perfusion are slightly different according to different TIC analysis software. Generally, blood perfusion quantitative parameters mainly include PI (peak intensity, the maximum intensity of the time-intensity curve during bolus transit), TTP (time to peak, time needed to reach peak intensity), RT (rise time, the difference between TTP and the time the first microbubble reached the lesion), MTT (mean transit time, circulation time of contrast agent in the area under investigation), WIS (wash-in slope, the ratio of PI to TTP), AS (ascending slope, the slope of the ascending branch of TIC, which showed mean perfusion speed after presence of the contrast agent and reflected local tissue perfusion rate), AUC and so on. TIC analyses of BC usually show shortening of RT and TTP and increasing PI and AS. Chemotherapy causes cytotoxic tumor cell death, resulting in reduced tissue vascular endothelial growth factor levels, apoptosis of immature endothelial cells with secondary vascular shutdown, and decreased blood perfusion, which in turn leads to slower wash-in of contrast agents. ${ }^{48}$ However, a poor response to $\mathrm{NAC}$ results in ongoing production of angiogenic factors that might maintain or increase the proportion of immature vessels. $^{49,50}$ According to the changes in blood vessels in the tumor after NAC PCR, PI, ${ }^{16,40,49} \mathrm{WIS}^{40}{ }^{4 \mathrm{AS}}{ }^{16}$ and AUC $^{51}$ were found to be significantly decreased, and TTP, ${ }^{40,43,49}$ MTT $^{49}$ and $\mathrm{RT}^{44}$ were significantly increased. Although multiple logistic regression analysis revealed that changes in quantitative parameters of TIC and diameter of the tumor were significant independent predictors of $\mathrm{pCR}$, the parameters of TIC, which are used as a functional technique to evaluate tumor response to $\mathrm{NAC}$, were superior and predicted earlier in response to NAC than changes in tumor size. ${ }^{49}$ In fact, placement of the regions of interest (ROIs) in CEUS research is also crucial for quantitative TIC analysis. ${ }^{52,53}$ TIC parameters were analyzed for different ROIs in Lee et al research. ${ }^{54}$ ROI 1, ROI 2, ROI 3, and ROI 4 targeted the hotspot area of greatest enhancement, area of hyperenhancement, entire tumor on grayscale ultrasound and normal parenchyma, respectively, and the results led to a recommendation for the less subjective ROI 2 or ROI 3. Studies have confirmed that breast CEUS combined with TIC curve analysis not only provides information about blood perfusion but also provides a variety of quantitative parameters that greatly improve the accuracy of predicting the response to NAC.

\section{Elastography for Assessing the Response to NAC}

Elastography, which is similar to palpation but more sensitive and objective, can assess tissue stiffness. Elastography is a recently developed, convenient and noninvasive imaging technique. There are two types of techniques in elastography: strain elastography and shear wave elastography. The strain elastography technique includes strain elastography (SE) and acoustic radiation force impulse (ARFI), while the shear wave elastography technique includes transient elastography (TE), point shear wave elastography (pSWE) and shear wave elastography (SWE). The most common methods that are widely used in the clinic are SE and SWE. In general, the ability of elastography to predict the efficacy of NAC was significantly higher than that of grayscale ultrasound and 
comparable to that of CEUS and MRI. ${ }^{42,55,56}$ The sensitivity, specificity, accuracy, PPV, NPV and AUC of predicting the efficacy of NAC by elastography were found to be $59-100 \%, 63-100 \%, 74-83 \%, 71-79 \%, 66-86 \%$ and $0.75-0.88$, respectively, in previous studies. ${ }^{42,56-59}$

SE is an imaging modality based on mechanical properties that uses manual compression and measures the degree of tissue deformation. ${ }^{60}$ The Tsukuba elasticity score (TES) can be used for scoring according to different colors, which represent the different stiffnesses of the tissue. ${ }^{61}$ In addition to the elastography score, the strain rate (SR) can be calculated by software that measures the strain rate ratio of elastography according to the ratio of stiffness between the lesion area and the surrounding normal tissue. ${ }^{62,63}$ Stiffer masses are more likely to represent malignancies. ${ }^{64}$ Hayashi et al first reported tumor stiffness assessed using SE. ${ }^{65}$ They examined the correlation between elasticity scores and treatment responses. The results demonstrated that stiffer tumors were less likely to exhibit clinical responses and pCR than more compliant tumors. However, other research demonstrated that SE imaging before NAC had no relationship with pathological complete response. ${ }^{64}$ The change in score and SR of elastography after NAC can be acquired by evaluating the score and SR of elastography before and after NAC. The score and SR of elastography of the tumors obtaining pCR were found to be decreased, and the degree of reduction was higher than those without $\mathrm{pCR} .{ }^{57,58} \mathrm{SE}$ can predict the efficacy of NAC earlier. Falou et al found that SE can predict the response of tumors to NAC in the medium term (4 weeks) by using SR, and the sensitivity and specificity were $100 \%{ }^{57}$ Fernandes et al demonstrated that SR can predict the pCR of tumors in the early stage $(2 \text { weeks })^{58}$ with a sensitivity and specificity of $84 \%$ and $85 \%$, respectively. Wang et al analyzed the parameters of CUES and SE before and after NAC for $\mathrm{BC}$, and the results showed that the AUCs of CEUS and SE in predicting the efficacy of NAC were 0.86 and 0.72 , respectively, and the predictive accuracies were $89 \%$ and $91 \%$, respectively. ${ }^{42}$ They indicated that CEUS and SE had equal advantages in predicting the response to NAC, which was the same conclusion as that of the $\mathrm{Gu}$ et al study. ${ }^{64}$ However, SE also has certain limitations. First, SE is highly dependent on the operators due to manual pressurization. As a result, human error can occur due to the difference in the measurement by different operators. Second, the elastography score is based on the visual observation of the color of the ROI, which is highly subjective and may also introduce human error.

SWE, which uses acoustic radiation force to introduce a disturbance and measures the speed of propagation of shear waves, can be used in conjunction with Hooke's law to derive the Young's modulus of tissue. ${ }^{60,66,67}$ The parameter maximum stiffness (Emax), mean stiffness (Emean), and standard deviation (SD), where $\mathrm{E}$ is defined by Young's modulus and measured in kilopascals and SD gives an indication of heterogeneity, refer to the elasticity values of the tissue within an ROI outlined on the US image. High mean stiffness values are more likely to be malignant. ${ }^{66-70}$ SWE can play a complementary role to conventional US. ${ }^{66,67}$ Stiffer tumors were found to be less likely to exhibit clinical responses and $\mathrm{pCR} .{ }^{71}$ Young's modulus of tumors with pCR after NAC was lower than that of tumors without $\mathrm{pCR} .{ }^{59}$ The change in Young's modulus before and after NAC could be calculated, and the larger the change was, the more likely was the indication of a response to NAC. ${ }^{56,59,72} \mathrm{SWE}$ is also believed to be an effective early predictor of pCR after NAC. Evans et al demonstrated that the changes in tumor stiffness after 3 weeks of NAC were closely related to $\mathrm{pCR} .{ }^{56}$ Jing et al showed that the response to NAC could be predicted by the change in Young's modulus two weeks after NAC, and the predictive sensitivity and specificity were $73 \%$ and $86 \%$, respectively. ${ }^{72}$ One advantage of SWE is that residual fibrous masses with no residual cancer tend to appear compliant in SWE, so that assessment by SWE is less prone to errors. However, there are a few limitations of SWE. It is difficult to assess deep lesions in BC patents, and the technique is influenced by patients' breathing. ${ }^{56}$

\section{Three-Dimensional (3D) Techniques for Assessing Response to NAC}

A 3D automated breast volume scanner (ABVS) is another novel and innovative imaging technique in breast ultrasound. ABVS is not only an observer-independent, automated and standardized method but also provides a large field of view using high-frequency transducers, and computer-aided detection software significantly reduces interpretation time. ${ }^{73}$ ABVS has the ability to calculate volume using 3D imaging software, and multiple studies have shown a higher correlation with histopathological tumor response than conventional US. ${ }^{73}$ van Egdom et al compared the efficacy of ABVS and MRI in predicting the efficacy of NAC. ${ }^{74}$ In the study, MRI and ABVS showed 
absolute concordance in $73 \%$ of patients for mid-NAC evaluation. Tumor response to NAC in midtreatment was evaluated using the product change in the two largest perpendicular diameters (PC) or the longest diameter change (LDC) in the study by Wang et al. ${ }^{75}$ All four prediction methods (PC on axial planes, LDC on axial planes, PC on coronal planes and LDC on coronal planes) displayed high AUCs (0.83-0.89), and the sensitivity, specificity, PPV, and NPV were 86-88\%, 62-85\%, 28-51\%, 96-98\%, respectively. ABVS was used to predict final pCR after 4 cycles of chemotherapy. The results demonstrated that the ABVS is a useful tool for the evaluation of pCR after NAC.

There are also additional 3D technologies applied in US, such as 3D power color US and 3D CEUS. Folkman et al emphasized the importance of angiogenesis in tumor growth. ${ }^{76}$ Vascular endothelial growth factors (VEGFs) are important regulatory cytokines, and microvessel density (MVD) has been the gold standard for assessing tumor angiogenesis. 3D power color flow with high-definition flow (HDF) technology facilitates the detection of vascular morphology imaging and better discriminates malignant breast tumors from benign lesions than 2D Doppler US. 3D power Doppler US provides high-resolution Doppler signal reflection and is not limited by the angle of the vessel, allowing 3D imaging of relevant vessels and observation of a tumor vascular mass by comparing perfusion changes and vessel density before and after NAC. ${ }^{77-79} 3 \mathrm{D}$ power Doppler US with HDF was considered to accurately predict response according to determination of changes in vascularity after NAC. The vascularization index (VI, the ratio between the color boxes and the total number of voxels in the volume of interest, representing the vessel density in the defined volume), flow index (FI, the mean energy of the voxel per color, representing the average intensity of flow), and vascularization-flow index (VFI, mean color value for all voxels in the volume, representing the intensity of both vascularization and flow) were extracted for estimation of vascularization and flow index. It was demonstrated that the most accurate prediction of pCR was achieved after the second chemotherapy treatment, with an accuracy of $88 \%$ and an AUC of $0.76 .{ }^{80}$ The combined advantages of CEUS and 3D-US, 3DCEUS, can evaluate tumor vascularity in a threedimensional field. In Jia's research, the 3D-CEUS score and DCE-MRI score were calculated according to the distribution and shape of breast tumor blood vessels in 3DCEUS, and the enhancement pattern and index of tumor blood vessels in DCE-MRI, MVD and VEGF were also calculated. ${ }^{81}$ The results showed that the 3D-CEUS score, DCE-MRI score, MVD and VEGF were significantly decreased after NAC. It was also shown that the efficacy of 3D-CEUS and DCE-MRI in predicting pCR after NAC was consistent. The sensitivity, specificity, PPV, NPV, and AUC were $88 \%$ and $88 \%, 100 \%$ and $100 \%, 100 \%$ and $100 \%, 98 \%$ and $98 \%$ and 0.93 and 0.99 , respectively, when the $3 \mathrm{D}$-CEUS score was $<1$ and $\triangle 3 \mathrm{D}$-CEUS score was $>6$ after NAC. The study also demonstrated that the 3D-CEUS score was significantly correlated with MVD and VEGF both before and after NAC, while DCE-MRI was not correlated with MVD or VEGF. These results showed that 3D-CEUS is effective for assessing the response to NAC. Although 3D power color US and 3DCEUS are rarely used in the clinic, they have a high accuracy in predicting the efficacy of NAC and are related to angiogenic factors, and 3D techniques still have certain development prospects.

\section{Other US Techniques for Assessing Response to NAC}

Changes in tumor echogenicity have also been applied to predict the response to NAC. Matsuda et al retrospectively examined 52 patients with triple-negative $\mathrm{BC}$ who received NAC and calculated changes in echogenicity. ${ }^{82}$ Pixel values are represented in black to white as different numeric values. It was demonstrated that the echogenicity changes (ratio and difference) in tumor and fat regions before and after NAC would predict pCR. The sensitivity, specificity and AUC were $70-74 \%, 81-82 \%$ and $0.78-0.80$, respectively. Dobruch-Sobczak et al found that the changes in the echogenicity of tumors after 3 courses of NAC exhibited the strongest statistical correlation with the percentage of residual malignant cells used in histopathology to assess the response to treatment (odds ratio $=60$ ). ${ }^{83}$ Changes in tumor echogenicity were demonstrated to predict response with satisfactory accuracy and may be considered for early NAC monitoring. Assessing changes in echogenicity is based on the backscattered spectrum of QUS. ${ }^{84}$ NAC can induce microstructural transformation, cell death, heterogeneous tumor morphology and edema. These cellular organizational changes alter US backscatter and increase tumor echogenicity. $^{85}$ Conventional US imaging involves "B-mode" images and loses much of the frequencydependent information with the conversion of radiofrequency (RF) data. Compared to conventional US, QUS 
Table I Accuracy, Sensitivity, Specificity, Accuracy, PPV, and NPV of Different Assessment Methods for the Prediction of Neoadjuvant Chemotherapy Response in Breast Cancer

\begin{tabular}{|c|c|c|c|c|c|}
\hline & $\operatorname{Sen}(\%)$ & Spe(\%) & Accuracy $(\%)$ & PPV(\%) & NPV(\%) \\
\hline Grayscale US & $36-61$ & $78-90$ & $7 I-74$ & $60-67$ & 29-75 \\
\hline CEUS & $85-96$ & $78-87$ & $84-86$ & 92 & 78 \\
\hline Elastography & $59-100$ & $63-100$ & $74-83$ & $71-79$ & $66-86$ \\
\hline ABVS & $86-88$ & $62-85$ & - & $28-51$ & $96-98$ \\
\hline 3D power Doppler US(HDF) & - & - & 88 & - & - \\
\hline 3D-CEUS & 88 & 100 & - & 100 & 98 \\
\hline QUS(ANN) & 89 & 85 & 87 & - & - \\
\hline DOT & 74 & 77 & 77 & 93 & 74 \\
\hline
\end{tabular}

Abbreviations: Sen, sensitivity; Spe, specificity; PPV, positive predictive value; NPV, negative predictive value; CEUS, contrast-enhanced ultrasound; ABVS, automated breast volume scanner; HDF, high-definition flow; QUS, quantitative ultrasound; ANN, artificial neural network; DOT, diffuse optical tomography.
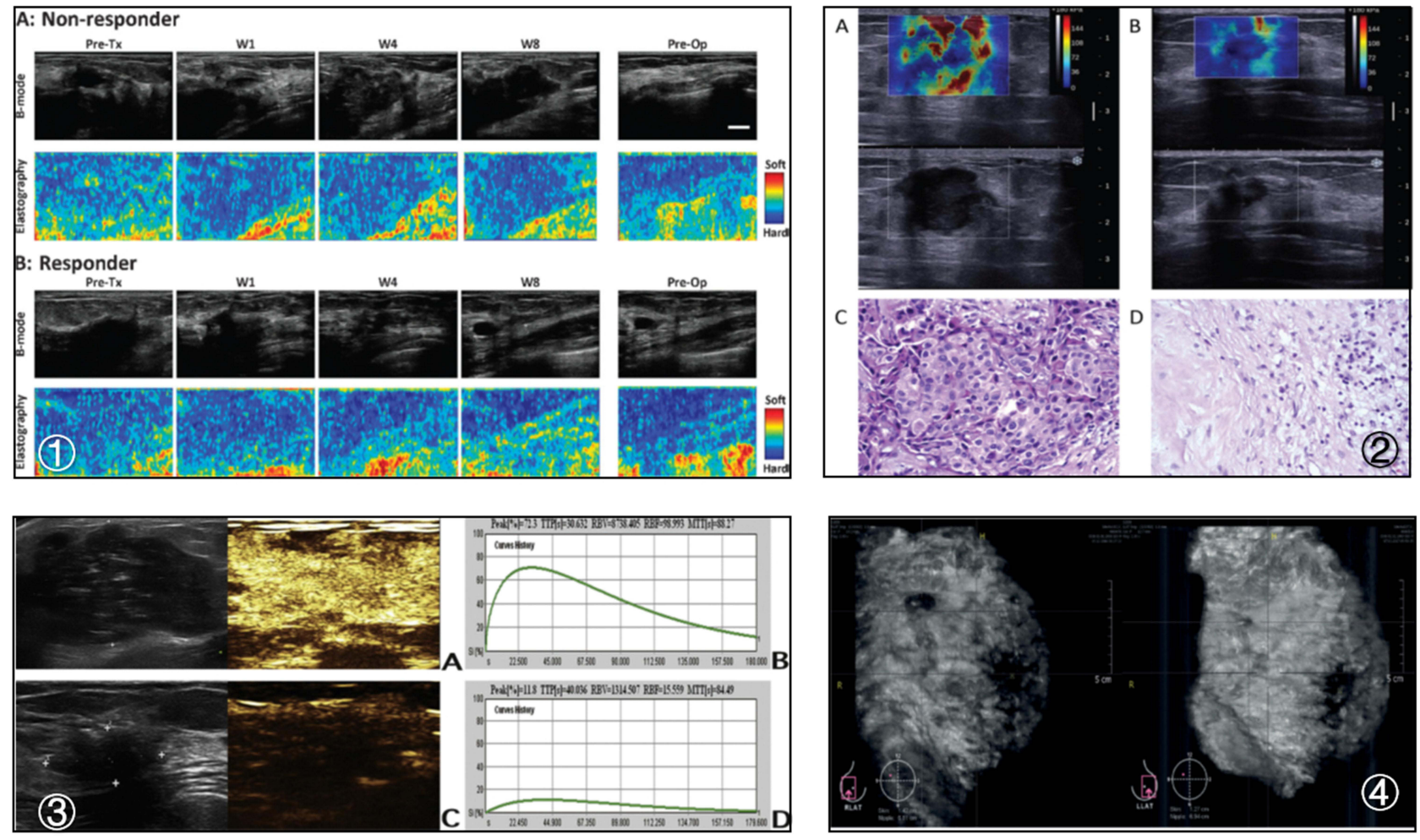

Figure I (1) Representative ultrasound B-mode images and elastography images for a nonresponder and a responder acquired before and after treatment. (A) Nonresponders typically exhibited no change in tumor elasticity during treatment for several weeks (average strain ratios ranged from $1.29 \pm 0.04$ before treatment to $1.23 \pm 0.11$ at week 8). (B) Responders exhibited visibly detectable changes in tumor stiffness (average strain ratios ranged from I.26 \pm 0.11 before treatment to I.04 \pm 0.05 at week 8). Reprinted from Translational Oncology, Vol 6/edition number I, Falou O, Sadeghi-Naini A, Prematilake S, et al, Evaluation of neoadjuvant chemotherapy response in women with locally advanced breast cancer using ultrasound elastography, Pages No. 17-24, Copyright (2013), with permission from Elsevier. ${ }^{57}$ (2) SWE and corresponding pathologic images from a complete response patient after NAC. (A) The mean tumor stiffness value as determined at baseline was $223.1 \mathrm{kPa}$. (B) After the second cycle of chemotherapy, the mean tumor stiffness value was $44.7 \mathrm{kPa}$, and $\Delta$-stiffness was $77.9 \%$. (C and $\mathbf{D}$ ) Pathological results before chemotherapy and after surgery. Reproduced with permission from Jing H, Cheng W, Li ZY, et al, Early evaluation of relative changes in tumor stiffness by shear wave elastography predicts the response to neoadjuvant chemotherapy in patients with breast cancer and John Wiley \& Sons, Inc. () 2016 by the American Institute of Ultrasound in Medicine. ${ }^{72}$ (3) CEUS and TIC images from a complete response patient after NAC. Before NAC, (A) grayscale US showed a hypoechoic lesion with an irregularly shaped and distinct margin. (A) A CEUS image obtained $39 \mathrm{~s}$ after contrast agent injection showed an inhomogeneous hyperenhancement lesion with an indistinct margin. (B) The TIC on CEUS shows strong and rapid perfusion. After NAC, (C) US showed a decrease in the tumor size. (C) CEUS showed hypo-enhancement. (D) The TIC at CEUS shows weak and slow enhancement. Reprinted from European Journal of Radiology, Vol I03, Wan CF, Liu XS, Wang L, Zhang J, Lu JS, Li FH, Quantitative contrast-enhanced ultrasound evaluation of pathological complete response in patients with locally advanced breast cancer receiving neoadjuvant chemotherapy, Pages No. 118-123, Copyright (2018), with permission from Elsevier ${ }^{49}$ (4) Example of the ABVS used for response evaluation. Frontal view (anterior-posterior) of the right breast. The left picture shows the tumor (upper left corner) pre-NAC. The right picture shows the same patients mid-NAC with a smaller tumor (upper left corner). Adapted from European Journal of Radiology, Vol 104, van Egdom LSE, Lagendijk M, Heijkoop EHM, et al, Threedimensional ultrasonography of the breast; An adequate replacement for MRI in neoadjuvant chemotherapy tumour response evaluation? - RESPONDER trial, Pages No. 94-100, Copyright (2018), with permission from Elsevier. ${ }^{74}$ 
imaging retains these RF data and displays the data as a frequency spectrum using a fast Fourier transform (FFT) algorithm. ${ }^{86}$ As a result, QUS was found to predict the response to NAC more accurately by supplying more information from US images. QUS uses variation in the acoustic property within tissues to characterize microstructural features and has been used in the detection of tumor response to chemotherapy of cancer. ${ }^{87}$ QUS parameters include MBF (midband fit), SS (spectral slope), SI (spectral intercept), ACE (attenuation coefficient estimate), SAS (spacing among scatterers), ASD (acoustic-scatterer diameter) and AAC (average acoustic-scatterer concentration). Sannachi et al analyzed QUS parameters and texture features extracted from QUS parameters. ${ }^{87}$ The accuracies ranged from $68 \%$ to $92 \%$ according to different multifeature response classification algorithms (a linear discriminant analysis (LDA), a k-nearest-neighbor classifier (KNN), and a radial-basisfunction support vector machine classifier (SVM-RBF)) to differentiate treatment responders. Tadayyon et al analyzed the capacity for predicting the response to NAC with QUS and an artificial neural network (ANN) classifier. ${ }^{88}$ They found that the sensitivity, specificity, accuracy, and AUC of the QUS model with an ANN classifier for predicting response were $89 \%, 85 \%, 87 \%$, and 0.90 , respectively.
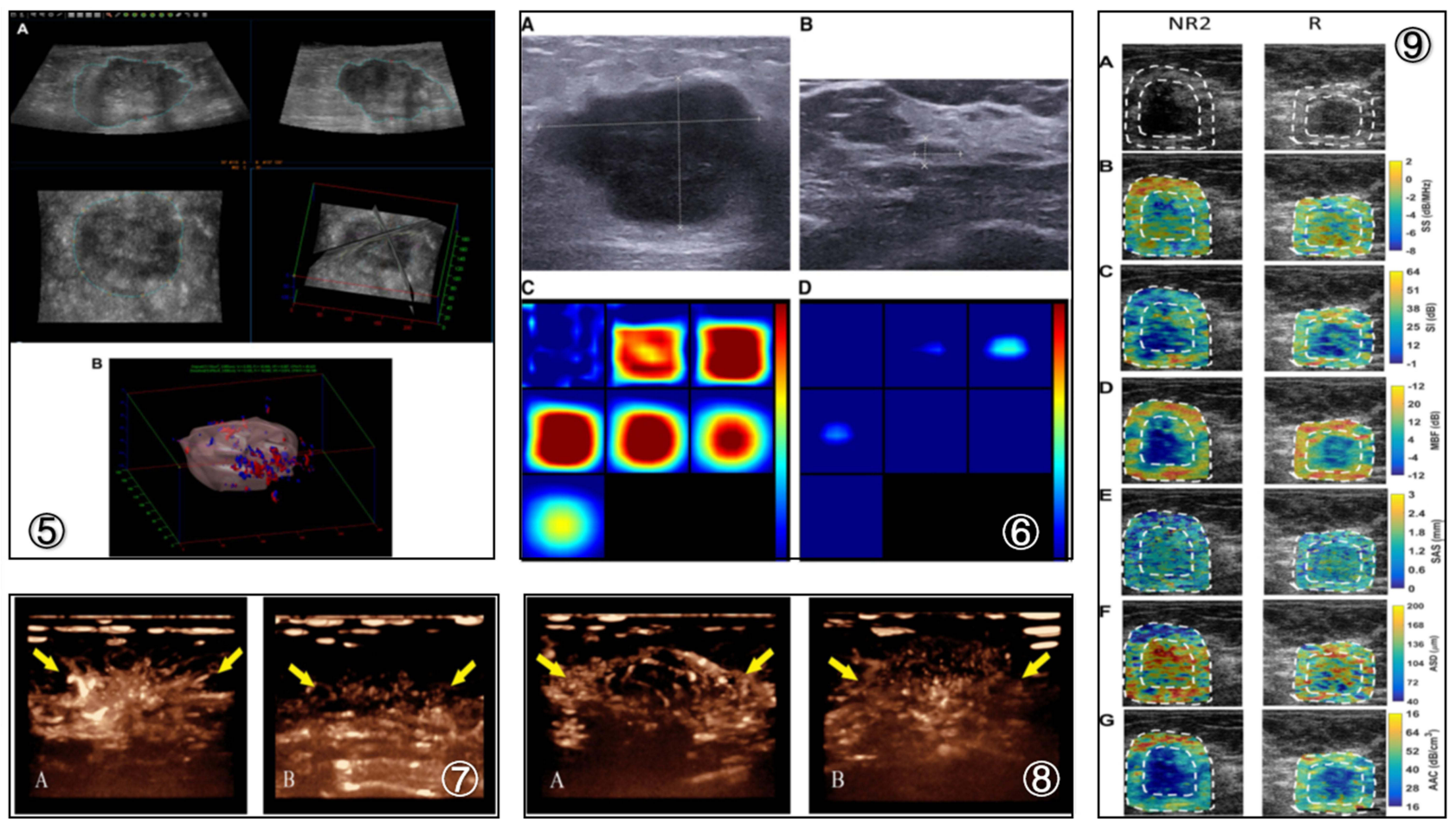

Figure 2 (5) Tumor margin obtained on 3D-HDF US images and an established 3D contour. (A) The tumor margin was sketched on a 2D slice B-mode US image, and the 3D contours were then established. The sketched margin is indicated with a blue dotted line. (B) Full image of 3D contours and vascular flow direction of the tumor. Reproduced from Shia WC, Huang YL, Wu HK, Chen DR, Using flow characteristics in three-dimensional power Doppler ultrasound imaging to predict complete responses in patients undergoing neoadjuvant chemotherapy and John Wiley and Sons. (C) 2017 by the American Institute of Ultrasound in Medicine. ${ }^{80}$ (6) Image of a responder case obtained by using ultrasound-guided diffuse optical tomography. (A and B) US images show US measuring a presize of $3.6 \mathrm{~cm}$ and a postsize of $0.7 \mathrm{~cm}$ in diameter. (C and $\mathbf{D}$ ) Pre- and posttreatment reconstructed optical absorption maps show that the lesion was resolved in slices from I (top left, left to right) to 7 (bottom left, left to right) and from 2 to 4 (top row, left to right). The pre- and posttreatment THCs were $279.0 \mu \mathrm{mol} / \mathrm{L}$ and I $28.0 \mu \mathrm{mol} / \mathrm{L}$, respectively. The vertical color scale from blue to red is the THC in micromoles per liter from low to high. Reprinted from Translational Oncology, Vol I l/edition number I, Zhi W, Liu G, Chang C, et al, Predicting treatment response of breast cancer to neoadjuvant chemotherapy using ultrasound-guided diffuse optical tomography, Pages No.56-64, Copyright (20I8), with permission from Elsevier. ${ }^{91}$ (7) Changes in peripheral vessels before and after completion of NAC in breast cancer patients displayed by 3D-CEUS. (A) Before NAC, the 3D-CEUS image showed coarse peripheral vessels with radial distribution (arrow) at the strongest stage of perfusion. (B) After completion of NAC, radial peripheral vessels (arrow) disappeared at the strongest stage of perfusion. Reproduced from Jia WR, Tang L,Wang DB, et al. Three-dimensional contrast-enhanced ultrasound in response assessment for breast cancer: a comparison with dynamic contrast-enhanced magnetic resonance imaging and pathology. Sci Rep. 2016;6:33832. ${ }^{81}$ (8) Changes in rim perfusion displayed by 3D-CEUS before and after completion of NAC for another breast cancer patient. (A) Before NAC, 3D-CEUS image showed coarse rim perfusion (arrow) at the strongest stage of perfusion. (B) After completion of NAC, rim perfusion (arrow) disappeared at the strongest stage of perfusion. Reproduced from Jia WR, Tang L,Wang DB, et al. Three-dimensional contrast-enhanced ultrasound in response assessment for breast cancer: a comparison with dynamic contrast-enhanced magnetic resonance imaging and pathology. Sci Rep. 2016;6:33832. ${ }^{81}$ (9) QUS images from a representative non-responder (NR2) patient and a representative responder patient with outlines of core and margin ROls. (A) B-mode images, (B) SS image, (C) SI images, (D) MBF images, (E) SAS images, (F) ASD, and (G) AAC images obtained prior to chemotherapy treatment initiation. Reproduced from Tadayyon H, Gangeh M, Sannachi L, et al. A priori prediction of breast tumour response to chemotherapy using quantitative ultrasound imaging and artificial neural networks. Oncotarget. 2019;10 (39):3910-3923. ${ }^{88}$ 
Changes in QUS parameters, particularly ultrasound backscatter intensity-based parameters, could distinguish responders and nonresponders at a later stage (week 8), while texture features could distinguish responders and nonresponders at early stages (weeks 1 and 4). The authors demonstrated an early-stage treatment response prediction model developed by combining QUS with texture analysis. ${ }^{87}$ Another study by Piotrzkowska-Wróblewska et al demonstrated that an integrated backscatter marker of QUS can better characterize the tumor pathological response and at an earlier stage (after the second and third NAC courses) of therapy with AUCs of 0.69 and 0.82 , respectively. ${ }^{89}$

Recently, it was reported that the NIR technique utilizes intrinsic hemoglobin contrast, which is directly related to tumor angiogenesis and has shown great potential for evaluating tumor vasculature and oxygen consumption responses to NAC by optical tomography and optical spectroscopy. ${ }^{90}$ DOT is an optical imaging technique that uses near-infrared light to probe the absorption and scattering properties of biological tissues and to acquire information on tumor physiology, biochemistry, angiogenesis, and hypoxia. ${ }^{91-95}$ DOT combined with US has been explored for breast cancer diagnosis and monitoring NAC responses in $\mathrm{BC}$. Combining pretreatment hemoglobin content and hemoglobin changes measured at early treatment cycles with standard pathological variables improves the predictive accuracy of NAC. ${ }^{96}$ The size and total hemoglobin concentration (THC) of the lesions were measured 1 day before biopsy and 1 to 2 days before surgery to predict $\mathrm{BC}$ response to NAC using US-guided DOT in the research of Zhi et al. ${ }^{91}$ The sensitivity, specificity, accuracy, PPV, NPV, and AUC were $74 \%$ and $80 \%, 77 \%$ and $53 \%, 77 \%$ and $38 \%, 93 \%$ and $88 \%, 74 \%$ and $77 \%, 0.75$ and 0.69 , respectively, when $\triangle \mathrm{THC}$ was $23.9 \%$ and $\triangle$ SIZE was $42.6 \%$. Moreover, $\triangle \mathrm{THC}$ and $\triangle$ SIZE can be used for response evaluation and earlier prediction of the response after three rounds of NAC. The authors considered US-guided DOT to be useful for early evaluation and prediction of the response to NAC.

The sensitivity, specificity, accuracy, PPV, and NPV of different US assessment methods for the prediction of neoadjuvant chemotherapy response in $\mathrm{BC}$ according to the results of the literature review are shown in Table 1. In addition, Figures 1 and 2 show US manifestations of different assessment methods in BC after NAC.

\section{Conclusions}

The goals of NAC have anecdotally been to enable breast conservation and prognostic information. US enables the advantages of portability, low cost, convenient follow-up detection without radiation emission and so on. In general, grayscale US has a low accuracy in the evaluation of the efficacy of NAC, while CEUS, elastography, 3D techniques, and other US techniques (QUS, DOT) can improve the accuracy of prediction. US technology combined with functional examination would be a great development prospect in assessing the response to NAC, and US is expected to be increasingly accepted to predict the efficacy of NAC in the future. However, this paper reviewed only the changes in $\mathrm{BC}$ mass after NAC by US and did not examine changes in lymph nodes in the corresponding region. A more detailed review would incorporate the changes in $\mathrm{BC}$ masses and lymph nodes observed by US after NAC.

\section{Funding}

The National Key R\&D Program of China (2018YFC0114900), the National Natural Science Foundation of China(81971623, the Major Research plan of the National Natural Science Foundation of China (91630311, the Natural Science Foundation of Zhejiang Province (SZ20H180002), Zhejiang Society Jiont Foundation for Mathematical Medicine(LSY19H180015), National S\&T Major Project of China (2018ZX10301201).

\section{Disclosure}

Jing Wang and Yanhua Chu are co-first authors. The authors report no conflicts of interest in this work.

\section{References}

1. Sung H, Ferlay J, Siegel RL, et al. Global cancer statistics 2020: GLOBOCAN estimates of incidence and mortality worldwide for 36 cancers in 185 countries. CA Cancer J Clin. 2021;71(3):209-249. doi: $10.3322 /$ caac. 21660

2. Gradishar WJ, Moran MS, Abraham J, et al. NCCN guidelines ${ }^{\circledR}$ insights: breast cancer, Version 4.2021. J Natl Compr Canc Netw. 2021;19(5):484-493.

3. Mamounas EP. Impact of neoadjuvant chemotherapy on locoregional surgical treatment of breast cancer. Ann Surg Oncol. 2015;22 (5):1425-1433. doi:10.1245/s10434-015-4406-6

4. Golshan M, Cirrincione CT, Sikov WM, et al. Impact of neoadjuvant chemotherapy in stage II-III triple negative breast cancer on eligibility for breast-conserving surgery and breast conservation rates: surgical results from CALGB 40603 (Alliance). Ann Surg. 2015;262 (3):434-439. doi:10.1097/SLA.0000000000001417

5. De La Cruz LM, McDonald ES, Mick R, et al. Anti-HER2 CD4+ T-helper type 1 immune response is superior to breast MRI for assessing response to neoadjuvant therapy in patients with HER2-positive breast cancer. Ann Surg Oncol. 2017;24(4):1057-1063. doi:10.1245/ s10434-016-5651-z

6. Taghizadeh Kermani A, Hosseini S, Fanipakdel A, et al. A randomized clinical trial on the antitumoral effects of low molecular weight heparin in the treatment of esophageal cancer. J Cell Physiol. 2019;234(4):4191-4199. doi:10.1002/jcp.27177 
7. Fazilat-Panah D, Vakili Ahrari Roudi S, Keramati A, et al. Changes in Cytokeratin 18 during neoadjuvant chemotherapy of breast cancer: a prospective study. Iran J Pathol. 2020;15(2):117-126. doi:10. 30699/ijp.2020.116238.2261

8. Homaei Shandiz F, Fanipakdel A, Forghani MN, et al. Clinical efficacy and side effects of IORT as tumor bed boost during breast-conserving surgery in breast cancer patients following neoadjuvant chemotherapy. Indian $J$ Gynecol Oncol. 2020;18:46. doi:10.1007/s40944-020-00389-5

9. Sedighi Pashaki A, Mohammadian K, Afshar S, et al. A randomized, controlled, parallel-group, trial on the effects of melatonin on fatigue associated with breast cancer and its adjuvant treatments. Integr Cancer Ther. 2021;20:1534735420988343. doi:10.1177/1534735420988343

10. Salek R, Dehghani M, Mohajeri SA, Talaei A, Fanipakdel A, Javadinia SA. Amelioration of anxiety, depression, and chemotherapy related toxicity after crocin administration during chemotherapy of breast cancer: a double blind, randomized clinical trial. Phytother Res. 2021;35:24.

11. Liu SV, Melstrom L, Yao K, Russell CA, Sener SF. Neoadjuvant therapy for breast cancer. J Surg Oncol. 2010;101(4):283-291. doi: $10.1002 /$ jso. 21446

12. Symmans WF, Peintinger F, Hatzis C, et al. Measurement of residual breast cancer burden to predict survival after neoadjuvant chemotherapy. J Clin Oncol. 2007;25(28):4414-4422. doi:10.1200/ JCO.2007.10.6823

13. Ogston KN, Miller ID, Payne S, et al. A new histological grading system to assess response of breast cancers to primary chemotherapy: prognostic significance and survival. Breast. 2003;12(5):320-327. doi:10.1016/S0960-9776(03)00106-1

14. Sheikhbahaei S, Trahan TJ, Xiao J, et al. FDG-PET/CT and MRI for evaluation of pathologic response to neoadjuvant chemotherapy in patients with breast cancer: a meta-analysis of diagnostic accuracy studies. Oncologist. 2016;21(8):931-939. doi:10.1634/theoncologist.2015-0353

15. Gu YL, Pan SM, Ren J, Yang ZX, Jiang GQ. Role of magnetic resonance imaging in detection of pathologic complete remission in breast cancer patients treated with neoadjuvant chemotherapy: a meta-analysis. Clin Breast Cancer. 2017;17(4):245-255. doi:10. 1016/j.clbc.2016.12.010

16. Amioka A, Masumoto N, Gouda N, et al. Ability of contrast-enhanced ultrasonography to determine clinical responses of breast cancer to neoadjuvant chemotherapy. Jpn J Clin Oncol. 2016;46(4):303-309. doi:10.1093/jjco/hyv215

17. Ma Y, Zhang S, Li J, Li J, Kang Y, Ren W. Comparison of strain and shear-wave ultrasounic elastography in predicting the pathological response to neoadjuvant chemotherapy in breast cancers. Eur Radiol. 2017;27(6):2282-2291. doi:10.1007/s00330-016-4619-5

18. Marinovich ML, Macaskill P, Irwig L, et al. Agreement between MRI and pathologic breast tumor size after neoadjuvant chemotherapy, and comparison with alternative tests: individual patient data meta-analysis. BMC Cancer. 2015;15:662. doi:10.1186/s12885-015$1664-4$

19. Eisenhauer EA, Therasse P, Bogaerts J, et al. New response evaluation criteria in solid tumours: revised RECIST guideline (version 1.1). Eur J Cancer. 2009;45(2):228-247. doi:10.1016/j.ejca.2008. 10.026

20. Hayashi M, Yamamoto Y, Iwase H. Clinical imaging for the prediction of neoadjuvant chemotherapy response in breast cancer. Chin Clin Oncol. 2020;9(3):31. doi:10.21037/cco-20-15

21. Dummin LJ, Cox M, Plant L. Prediction of breast tumor size by mammography and sonography-A breast screen experience. Breast. 2007;16(1):38-46. doi:10.1016/j.breast.2006.04.003

22. Hieken TJ, Harrison J, Herreros J, Velasco JM. Correlating sonography, mammography, and pathology in the assessment of breast cancer size. Am J Surg. 2001;182(4):351-354. doi:10.1016/S0002-9610(01) 00726-7
23. Gruber IV, Rueckert M, Kagan KO, et al. Measurement of tumor size with mammography, sonography and magnetic resonance imaging as compared to histological tumor size in primary breast cancer. $B M C$ Cancer. 2013;5(13):328. doi:10.1186/1471-2407-13-328

24. Haraldsdóttir KH, Jónsson $\mathrm{P}$, Halldórsdóttir AB, Tranberg KG, Ásgeirsson KS. Tumor size of invasive breast cancer on magnetic resonance imaging and conventional imaging (Mammogram/ Ultrasound): comparison with pathological size and clinical implications. Scand J Surg. 2017;106(1):68-73. doi:10.1177/ 1457496916631855

25. Vriens BE, de Vries B, Lobbes MB, et al. Ultrasound is at least as good as magnetic resonance imaging in predicting tumour size post-neoadjuvant chemotherapy in breast cancer. Eur $J$ Cancer. 2016;52:67-76. doi:10.1016/j.ejca.2015.10.010

26. Stein RG, Wollschläger D, Kreienberg R; BRENDA Study Group, et al. The impact of breast cancer biological subtyping on tumor size assessment by ultrasound and mammography - a retrospective multicenter cohort study of 6543 primary breast cancer patients. BMC Cancer. 16;2016:459. doi:10.1186/s12885-016-2426-7

27. Chagpar AB, Middleton LP, Sahin AA, et al. Accuracy of physical examination, ultrasonography, and mammography in predicting residual pathologic tumor size in patients treated with neoadjuvant chemotherapy. Ann Surg. 2006;243(2):257-264. doi:10.1097/01. sla.0000197714.14318.6f

28. Keune JD, Jeffe DB, Schootman M, Hoffman A, Gillanders WE, Aft RL. Accuracy of ultrasonography and mammography in predicting pathologic response after neoadjuvant chemotherapy for breast cancer. Am J Surg. 2010;199(4):477-484. doi:10.1016/j.amjsurg.2009.03.012

29. Choi JH, Lim HI, Lee SK, et al. The role of PET CT to evaluate the response to neoadjuvant chemotherapy in advanced breast cancer: comparison with ultrasonography and magnetic resonance imaging. J Surg Oncol. 2010;102(5):392-397. doi:10.1002/jso.21424

30. Swain SM, Sorace RA, Bagley CS, et al. Neoadjuvant chemotherapy in the combined modality approach of locally advanced nonmetastatic breast cancer. Cancer Res. 1987;47(14):3889-3894.

31. Cocconi G, Di Blasio B, Alberti G, Bisagni G, Botti E, Peracchia G. Problems in evaluating response of primary breast cancer to systemic therapy. Breast Cancer Res Treat. 1984;4(4):309-313. doi:10.1007/ BF01806044

32. Segel MC, Paulus DD, Hortobagyi GN. Advanced primary breast cancer: assessment at mammography of response to induction chemotherapy. Radiology. 1988;169(1):49-54. doi:10.1148/radiology. 169.1.3420282

33. Zhang K, Li J, Zhu Q, Chang C. Prediction of pathologic complete response by ultrasonography and magnetic resonance imaging after neoadjuvant chemotherapy in patients with breast cancer. Cancer Manag Res. 2020;12:2603-2612. doi:10.2147/CMAR.S247279

34. Schaefgen B, Mati M, Sinn HP, et al. Can routine imaging after neoadjuvant chemotherapy in breast cancer predict pathologic complete response? Ann Surg Oncol. 2016;23(3):789-795. doi:10.1245/ s10434-015-4918-0

35. Baumgartner A, Tausch C, Hosch S, et al. Ultrasound-based prediction of pathologic response to neoadjuvant chemotherapy in breast cancer patients. Breast. 2018;39:19-23. doi:10.1016/j.breast.2018.02.028

36. Wan CF, Du J, Fang H, Li FH, Zhu JS, Liu Q. Enhancement patterns and parameters of breast cancers at contrast-enhanced US: correlation with prognostic factors. Radiology. 2012;262(2):450-459. doi:10. 1148/radiol.11110789

37. Luo J, Chen JD, Chen Q, et al. Predictive model for contrast-enhanced ultrasound of the breast: is it feasible in malignant risk assessment of breast imaging reporting and data system 4 lesions? World J Radiol. 2016;8(6):600-609. doi:10.4329/wjr.v8.i6.600

38. Bernatik T, Becker D, Neureiter D, et al. Detection of liver metastases-comparison of contrast-enhanced ultrasound using first versus second generation contrast agents. Ultraschall Med. 2003;24 (3):175-179. doi:10.1055/s-2003-40060 
39. Lee SC, Grant E, Sheth P, et al. Accuracy of contrast-enhanced ultrasound compared with magnetic resonance imaging in assessing the tumor response after neoadjuvant chemotherapy for breast cancer. J Ultrasound Med. 2017;36(5):901-911. doi:10.7863/ ultra. 16.05060

40. Cao X, Xue J, Zhao B. Potential application value of contrast-enhanced ultrasound in neoadjuvant chemotherapy of breast cancer. Ultrasound Med Biol. 2012;38(12):2065-2071. doi:10.1016/j. ultrasmedbio.2012.07.027

41. Jia K, Li L, Wu XJ, Hao MJ, Xue HY. Contrast-enhanced ultrasound for evaluating the pathologic response of breast cancer to neoadjuvant chemotherapy: a meta-analysis. Medicine. 2019;98(4):e14258. doi:10.1097/MD.0000000000014258

42. Wang B, Jiang T, Huang M, et al. Evaluation of the response of breast cancer patients to neoadjuvant chemotherapy by combined contrast-enhanced ultrasonography and ultrasound elastography. Exp Ther Med. 2019;17(5):3655-3663.

43. Saracco A, Szabó BK, Tánczos E, Bergh J, Hatschek T. Contrastenhanced ultrasound (CEUS) in assessing early response among patients with invasive breast cancer undergoing neoadjuvant chemotherapy. Acta Radiol. 2017;58(4):394-402. doi:10.1177/0284185116658322

44. Kim Y, Kim SH, Song BJ, et al. Early prediction of response to neoadjuvant chemotherapy using dynamic contrast-enhanced MRI and ultrasound in breast cancer. Korean J Radiol. 2018;19(4):6 82-691. doi:10.3348/kjr.2018.19.4.682

45. Ricci P, Cantisani V, Ballesio L, et al. Benign and malignant breast lesions: efficacy of real time contrast-enhanced ultrasound vs. Magn Reson Imaging Ultraschall Med. 2007;28(1):57-62. doi:10.1055/ s-2006-927226

46. Fröhlich E, Muller R, Cui XW, Schreiber-Dietrich D, Dietrich CF. Dynamic contrast-enhanced ultrasound for quantification of tissue perfusion. J Ultrasound Med. 2015;34(2):179-196. doi:10.7863/ ultra.34.2.179

47. Hudson JM, Williams R, Tremblay-Darveau C, et al. Dynamic contrast enhanced ultrasound for therapy monitoring. Eur J Radiol. 2015;84(9):1650-1657. doi:10.1016/j.ejrad.2015.05.013

48. Darland DC, D'Amore PA. Blood vessel maturation: vascular development comes of age. J Clin Invest. 1999;103(2):157-158. doi:10. $1172 / \mathrm{JCI} 6127$

49. Wan CF, Liu XS, Wang L, Zhang J, Lu JS, Li FH. Quantitative contrast-enhanced ultrasound evaluation of pathological complete response in patients with locally advanced breast cancer receiving neoadjuvant chemotherapy. Eur $J$ Radiol. 2018;103:118-123. doi:10.1016/j.ejrad.2018.04.005

50. Mayr NA, Yuh WT, Arnholt JC, et al. Pixel analysis of MR perfusion imaging in predicting radiation therapy outcome in cervical cancer. $J$ Magn Reson Imaging. 2000;12(6):1027-1033. doi:10.1002/15222586(200012)12:6<1027::AID-JMRI31>3.0.CO;2-5

51. Dong T. Early response assessed by contrast-enhanced ultrasound in breast cancer patients undergoing neoadjuvant chemotherapy. Ultrasound $Q$. 2018;34(2):84-87. doi:10.1097/RUQ.0000000000000333

52. Nakata $\mathrm{N}$, Ohta $\mathrm{T}$, Nishioka $\mathrm{M}$, et al. Optimization of region of interest drawing for quantitative analysis: differentiation between benign and malignant breast lesions on contrast-enhanced sonography. J Ultrasound Med. 2015;34(11):1969-1976. doi:10. 7863/ultra.14.10042

53. Atri M, Hudson JM, Sinaei M, et al. Impact of acquisition method and region of interest placement on inter-observer agreement and measurement of tumor response to targeted therapy using dynamic contrast-enhanced ultrasound. Ultrasound Med Biol. 2016;42 (3):763-768. doi:10.1016/j.ultrasmedbio.2015.11.005

54. Lee YJ, Kim SH, Kang BJ, Kim YJ. Contrast-enhanced ultrasound for early prediction of response of breast cancer to neoadjuvant chemotherapy. Ultraschall Med. 2019;40(2):194-204. doi:10.1055/ a-0637-1601
55. Evans A, Whelehan P, Thompson A, et al. Identification of pathological complete response after neoadjuvant chemotherapy for breast cancer: comparison of greyscale ultrasound, shear wave elastography, and MRI. Clin Radiol. 2018;73(10):910.e1-910.e6. doi:10.1016/j. crad.2018.05.030

56. Evans A, Whelehan P, Thompson A, et al. Prediction of pathological complete response to neoadjuvant chemotherapy for primary breast cancer comparing interim ultrasound, shear wave elastography and MRI. Ultraschall Med. 2018;39(4):422-431. doi:10.1055/s-0043111589

57. Falou O, Sadeghi-Naini A, Prematilake S, et al. Evaluation of neoadjuvant chemotherapy response in women with locally advanced breast cancer using ultrasound elastography. Transl Oncol. 2013;6 (1):17-24. doi:10.1593/tlo.12412

58. Fernandes J, Sannachi L, Tran WT, et al. Monitoring breast cancer response to neoadjuvant chemotherapy using ultrasound strain elastography. Transl Oncol. 2019;12(9):1177-1184. doi:10.1016/j. tranon.2019.05.004

59. Lee SH, Chang JM, Han W, et al. Shear-wave elastography for the detection of residual breast cancer after neoadjuvant chemotherapy. Ann Surg Oncol. 2015;22(Suppl 3):S376-S384. doi:10.1245/s10434015-4828-1

60. Chang JM, Won JK, Lee KB, Park IA, Yi A, Moon WK. Comparison of shear-wave and strain ultrasound elastography in the differentiation of benign and malignant breast lesions. Am $J$ Roentgenol. 2013;201(2):W347-W356. doi:10.2214/AJR.12.10416

61. Itoh A, Ueno E, Tohno E, et al. Breast disease: clinical application of US elastography for diagnosis. Radiology. 2006;239(2):341-350. doi:10.1148/radiol.2391041676

62. Gennisson JL, Deffieux T, Fink M, Tanter M. Ultrasound elastography: principles and techniques. Diagn Interv Imaging. 2013;94 (5):487-495. doi:10.1016/j.diii.2013.01.022

63. Sigrist RMS, Liau J, Kaffas AE, Chammas MC, Willmann JK. Ultrasound elastography: review of techniques and clinical applications. Theranostics. 2017;7(5):1303-1329. doi:10.7150/thno.18650

64. Gu LS, Zhang R, Wang Y, et al. Characteristics of contrast-enhanced ultrasonography and strain elastography of locally advanced breast cancer. J Thorac Dis. 2019;11(12):5274-5289. doi:10.21037/jtd. 2019.11.52

65. Hayashi M, Yamamoto Y, Ibusuki M, et al. Evaluation of tumor stiffness by elastography is predictive for pathologic complete response to neoadjuvant chemotherapy in patients with breast cancer. Ann Surg Oncol. 2012;19(9):3042-3049. doi:10.1245/s10434-012-2343-1

66. Ricci P, Maggini E, Mancuso E, Lodise P, Cantisani V, Catalano C. Clinical application of breast elastography: state of the art. Eur J Radiol. 2014;83(3):429-437. doi:10.1016/j.ejrad.2013.05.007

67. Cosgrove DO, Berg WA, Doré CJ, et al. Shear wave elastography for breast masses is highly reproducible. Eur Radiol. 2012;22 (5):1023-1032. doi:10.1007/s00330-011-2340-y

68. Chang JM, Moon WK, Cho N, et al. Clinical application of shear wave elastography (SWE) in the diagnosis of benign and malignant breast diseases. Breast Cancer Res Treat. 2011;129(1):89-97. doi:10.1007/s10549-011-1627-7

69. Evans A, Whelehan P, Thomson K, et al. Invasive breast cancer: relationship between shear-wave elastographic findings and histologic prognostic factors. Radiology. 2012;263(3):673-677. doi:10.1148/ radiol.12111317

70. Chang JM, Park IA, Lee SH, et al. Stiffness of tumours measured by shear-wave elastography correlated with subtypes of breast cancer. Eur Radiol. 2013;23(9):2450-2458. doi:10.1007/s00330013-2866-2

71. Evans A, Armstrong S, Whelehan P, et al. Can shear-wave elastography predict response to neoadjuvant chemotherapy in women with invasive breast cancer? Br J Cancer. 2013;109(11):2798-2802. doi:10.1038/bjc.2013.660 
72. Jing H, Cheng W, Li ZY, et al. Early evaluation of relative changes in tumor stiffness by shear wave elastography predicts the response to neoadjuvant chemotherapy in patients with breast cancer. J Ultrasound Med. 2016;35(8):1619-1627. doi:10.7863/ultra.15.08052

73. Wojcinski S, Farrokh A, Hille U, et al. The Automated Breast Volume Scanner (ABVS): initial experiences in lesion detection compared with conventional handheld B-mode ultrasound: a pilot study of 50 cases. Int J Womens Health. 2011;3:337-346. doi:10.2147/IJWH.S23918

74. van Egdom LSE, Lagendijk M, Heijkoop EHM, et al. Threedimensional ultrasonography of the breast; An adequate replacement for MRI in neoadjuvant chemotherapy tumour response evaluation? RESPONDER trial. Eur J Radiol. 2018;104:94-100. doi:10.1016/j. ejrad.2018.05.005

75. Wang X, Huo L, He Y, et al. Early prediction of pathological outcomes to neoadjuvant chemotherapy in breast cancer patients using automated breast ultrasound. Chin J Cancer Res. 2016;28 (5):478-485. doi:10.21147/j.issn.1000-9604.2016.05.02

76. Folkman J, Merler E, Abernathy C, Williams G. Isolation of a tumor factor responsible for angiogenesis. $J$ Exp Med. 1971;133 (2):275-288. doi:10.1084/jem.133.2.275

77. Lee SW, Choi HY, Baek SY, Lim SM. Role of color and power Doppler imaging in differentiating between malignant and benign solid breast masses. J Clin Ultrasound. 2002;30(8):459-464. doi:10.1002/jcu. 10100

78. Demi L, Verweij MD, Van Dongen KW. Parallel transmit beamforming using orthogonal frequency division multiplexing applied to harmonic imaging-a feasibility study. IEEE Trans Ultrason Ferroelectr Freq Control. 2012;59(11):2439-2447. doi:10.1109/TUFFC.2012.2476

79. Chang RF, Huang SF, Moon WK, Lee YH, Chen DR. Computer algorithm for analysing breast tumor angiogenesis using 3-D power Doppler ultrasound. Ultrasound Med Biol. 2006;32(10):1499-1508. doi:10.1016/j.ultrasmedbio.2006.05.029

80. Shia WC, Huang YL, Wu HK, Chen DR. Using flow characteristics in three-dimensional power Doppler ultrasound imaging to predict complete responses in patients undergoing neoadjuvant chemotherapy. J Ultrasound Med. 2017;36(5):887-900. doi:10.7863/ultra.16.02078

81. Jia WR, Tang L, Wang DB, et al. Three-dimensional contrast-enhanced ultrasound in response assessment for breast cancer: a comparison with dynamic contrast-enhanced magnetic resonance imaging and pathology. Sci Rep. 2016;6:33832. doi:10.1038/srep33832

82. Matsuda N, Kida K, Ohde S, et al. Change in sonographic brightness can predict pathological response of triple-negative breast cancer to neoadjuvant chemotherapy. Breast Cancer. 2018;25(1):43-49. doi:10.1007/s12282-017-0782-z

83. Dobruch-Sobczak K, Piotrzkowska-Wróblewska H, Klimonda Z, Roszkowska-Purska K, Litniewski J. Ultrasound echogenicity reveals the response of breast cancer to chemotherapy. Clin Imaging. 2019;55:41-46. doi:10.1016/j.clinimag.2019.01.021

84. Alam SK, Feleppa EJ, Rondeau M, Kalisz A, Garra BS. Ultrasonic multi-feature analysis procedure for computer-aided diagnosis of solid breast lesions. Ultrason Imaging. 2011;33(1):17-38. doi:10.1177/ 016173461103300102
85. Sadeghi-Naini A, Papanicolau N, Falou O, et al. Quantitative ultrasound evaluation of tumor cell death response in locally advanced breast cancer patients receiving chemotherapy. Clin Cancer Res. 2013;19(8):2163-2174. doi:10.1158/1078-0432.CCR12-2965

86. DiCenzo D, Quiaoit K, Fatima K, et al. Quantitative ultrasound radiomics in predicting response to neoadjuvant chemotherapy in patients with locally advanced breast cancer: results from multi-institutional study. Cancer Med. 2020;9(16):5798-5806. doi:10.1002/cam4.3255

87. Sannachi L, Gangeh M, Tadayyon H, et al. Breast cancer treatment response monitoring using quantitative ultrasound and texture analysis: comparative analysis of analytical models. Transl Oncol. 2019;12 (10):1271-1281.

88. Tadayyon H, Gangeh M, Sannachi L, et al. A priori prediction of breast tumour response to chemotherapy using quantitative ultrasound imaging and artificial neural networks. Oncotarget. 2019;10 (39):3910-3923. doi:10.18632/oncotarget.26996

89. Piotrzkowska-Wróblewska H, Dobruch-Sobczak K, Klimonda Z, et al. Monitoring breast cancer response to neoadjuvant chemotherapy with ultrasound signal statistics and integrated backscatter. PLoS One. 2019;14(3):e0213749. doi:10.1371/journal.pone.0213749

90. Choe R, Durduran T. Diffuse optical monitoring of the neoadjuvant breast cancer therapy. IEEE J Sel Top Quantum Electron. 2012;18 (4):1367-1386. doi:10.1109/JSTQE.2011.2177963

91. Zhi W, Liu G, Chang C, et al. Predicting treatment response of breast cancer to ne foadjuvant chemotherapy using ultrasound-guided diffuse optical tomography. Transl Oncol. 2018;11(1):56-64. doi:10.1016/j.tranon.2017.10.011

92. Tromberg BJ, Cerussi A, Shah N, et al. Imaging in breast cancer: diffuse optics in breast cancer: detecting tumors in pre-menopausal women and monitoring neoadjuvant chemotherapy. Breast Cancer Res. 2005;7(6):279-285. doi:10.1186/bcr1358

93. Zhu Q, Cronin EB, Currier AA, et al. Benign versus malignant breast masses: optical differentiation with US-guided optical imaging reconstruction. Radiology. 2005;237(1):57-66. doi:10.1148/radiol.23 71041236

94. Fournier LS, Vanel D, Athanasiou A, et al. Dynamic optical breast imaging: a novel technique to detect and characterize tumor vessels. Eur J Radiol. 2009;69(1):43-49. doi:10.1016/j.ejrad.2008.07.038

95. Ueda S, Nakamiya N, Matsuura K, et al. Optical imaging of tumor vascularity associated with proliferation and glucose metabolism in early breast cancer: clinical application of total hemoglobin measurements in the breast. BMC Cancer. 2013;31(13):514. doi:10.1186/ 1471-2407-13-514

96. Xu C, Vavadi H, Merkulov A, et al. Ultrasound-guided diffuse optical tomography for predicting and monitoring neoadjuvant chemotherapy of breast cancers: recent progress. Ultrason Imaging. 2016;38(1):5-18.
Cancer Management and Research

\section{Publish your work in this journal}

Cancer Management and Research is an international, peer-reviewed open access journal focusing on cancer research and the optimal use of preventative and integrated treatment interventions to achieve improved outcomes, enhanced survival and quality of life for the cancer patient.
Dovepress

The manuscript management system is completely online and includes a very quick and fair peer-review system, which is all easy to use. Visit http://www.dovepress.com/testimonials.php to read real quotes from published authors. 